\title{
Circulatory adjustments after birth: effects on ventricular septal defect
}

\author{
Abraham M. Rudolph ${ }^{1}$ \\ From the Cardiovascular Research Institute and Department of Pediatrics, University of \\ California, San Francisco, California 94122, U.S.A.
}

The haemodynamic effects and clinical manifestations of congenital heart lesions may be greatly influenced by developmental changes in the circulation. The sequential changes associated with abnormal communications between the systemic and pulmonary circulations are well typified by the effect on ventricular septal defect. The possible influences of ventricular septal defect on the foetal circulation are first presented, and the interrelationship between ventricular septal defect and postnatal adjustments of the circulation are discussed.

The foetal circulation is characterized by the presence of essentially equal pressures in the aorta and pulmonary artery owing to the large communication afforded by the ductus arteriosus. Distribution of blood flow to the lungs as well as organs supplied by the systemic circulation is, therefore, determined by local vascular resistance. In the foetus the placental vascular bed has a relatively low resistance, and it receives about 40-50 per cent of the combined output of the two ventricles. The lung, however, has a high resistance to flow and receives only about 5 per cent of the combined ventricular output (Rudolph and Heymann, 1967). In the normal foetus, superior vena caval blood passes directly through the tricuspid valve into the right ventricle and pulmonary artery. Apart from the small proportion which enters the lungs, most of this blood is directed through the ductus arteriosus to the descending aorta. Inferior vena caval blood has a considerably higher $\mathrm{Po}_{2}$ than superior vena caval blood, since it includes the more highly oxygenated blood returning through the umbilical veins. A large proportion of inferior vena caval return crosses the foramen ovale into the left atrium to the left ventricle, and is thus distributed to the coronary and cerebral circulations. The $\mathrm{Po}_{2}$ of blood supplying the heart, brain, head, and neck is higher by $4-5 \mathrm{~mm}$. than that of blood in the descending aorta.

\footnotetext{
Ventricular septal defect in the foetus The presence of a ventricular septal defect in the foetus would not be expected to affect the circulation to any considerable extent. Since 1 Supported by a grant from the National Institutes of Health.
}

the pulmonary vascular resistance is high, blood reaching the right ventricle may be ejected through the defect into the left ventricle and then into the aorta. The difference in $\mathrm{PO}_{2}$ between the ascending aorta and descending aorta could be decreased. The evidence at present is that this would not materially alter the foetal growth and development or responses of the foetal circulation. This is based on the fact that transposition of the great arteries, in which there would be reversal of the normal ascending aortic to descending aortic $\mathrm{Po}_{2}$ difference, does not appear to affect normal development of the foetus.

\section{Circulatory adjustments after birth}

The elimination of the placental circulation removes a low resistance circuit which in the term foetus receives about 40-50 per cent of the combined ventricular output. There is therefore a conspicuous increase in the systemic vascular resistance associated with cutting or clamping of the umbilical cord.

The blood ejected by the right ventricle into the main pulmonary artery in the foetus courses mainly through the ductus arteriosus, and pulmonary vascular resistance is very high. It was suggested by Reynolds (1956) that the high pulmonary vascular resistance in the foetus was due to tortuosity and kinking of the small vessels in the unexpanded foetal lung. He further suggested that expansion of the lungs uncoiled these vessels, resulting in a decrease in their resistance to blood flow, thus explaining the prominent increase in pulmonary blood flow after birth.

The pulmonary blood vessels of the foetal and newborn animal are extremely sensitive 
to changes in $\mathrm{H}^{+}$ion concentration and $\mathrm{Po}_{2}$ to which they are exposed (Rudolph and Yuan, I966). The low $\mathrm{PO}_{2}$ in the pulmonary arteries of the foetus is thus probably largely responsible for the high pulmonary vascular resistance and also for the development of a thick medial muscular layer.

Physical expansion of the lungs by air at the time of birth may have a relatively minor influence in decreasing pulmonary vascular resistance (Cassin et al., 1964), but the major reduction is due to the rise in $\mathrm{Po}_{2}$ to which the pulmonary vessels are subjected when the alveoli are filled with air. Most blood vessels in the body are dilated by local hypoxia and constricted by oxygen, but the reason for the difference in the response of the pulmonary vessels has not been resolved. The possibility that the dilator effect of oxygen may not be direct but mediated through release of some chemical substance has been considered. Lloyd (1969) has shown that both carotid and pulmonary arterial segments constrict when surrounded by a cuff of lung tissue and subjected to hypoxia. These studies suggested that the response to oxygen is mediated through a substance released by lung parenchyma.

A further finding of interest is that expansion of the lungs of the foetal lamb with air or oxygen results in release of bradykinin in the pulmonary venous blood (Heymann et al., 1969). Bradykinin is a potent dilator of foetal pulmonary vessels (Campbell et al., 1968), and its formation in the lungs may be an important mechanism in the adaptations to birth.

The fall in pulmonary vascular resistance and rise in systemic resistance after birth results in a reversal of the relationships present in the foetus. While the ductus arteriosus is still patent the flow of blood through the ductus will change to a left-to-right shunt. Normally, however, the ductus arteriosus is functionally closed within IO-I 5 hours after birth, and pulmonary arterial pressure falls (Rudolph et al., 196I). There is then a continuing fall of pulmonary vascular resistance and pulmonary arterial pressure toward adult levels within a 2-3 week period. This is related to a thinning of the medial muscular layer of the small pulmonary vessels.

\section{Circulatory changes after birth and ven- tricular septal defect}

The haemodynamic and clinical features of ventricular septal defect are influenced by the circulatory changes after birth. The decrease in pulmonary vascular resistance after birth favours flow through the lungs versus the systemic circulation. This results in an increase in pulmonary venous return to the left atrium and ventricle. An increase in diastolic volume in the left ventricle will occur, and on the basis of the Frank-Starling mechanism left ventricular stroke volume rises. The magnitude of the left-to-right shunt will be determined by the size of the ventricular septal defect and the ratio of pulmonary to systemic vascular resistance.

As pulmonary vascular resistance falls after birth an increasing left-to-right shunt occurs, and if this is large, left ventricular failure develops with an increase in left ventricular enddiastolic pressure, left atrial and pulmonary venous pressures, and pulmonary congestion, and oedema ensues.

The response of the left ventricle to the increased volume load, and whether failure occurs or not, is influenced by several physiological factors. Hypertrophy of the left ventricle develops in response to the increased work load placed on it. If, therefore, the decline in pulmonary vascular resistance is gradual after birth, and therefore the increase in pulmonary blood flow is delayed, there may be adequate time for enough hypertrophy to develop so that cardiac failure does not become evident.

The sympathetic-adrenal system is most important in the adaptation of the circulation after birth in the presence of ventricular septal defect. It has been shown that blockade of beta-adrenergic receptors in dogs with induced left-to-right shunt lesions (artificial ductus arteriosus) will limit the size of the pulmonary venous return that can be tolerated without failure developing. Furthermore, acute cardiac failure which occurs when a large aorto-pulmonary shunt is established may be readily overcome by infusion of catecholamines (Rudolph, 1965).

Conditions which place an additional burden on the circulation may precipitate the development of failure in infants with ventricular septal defect. An infection may result in an increase in cardiac output, with an additional demand on the left ventricle, and induce cardiac failure. The normal decrease in haemoglobin level which occurs within the first 2-3 months after birth may be most important in the presence of a ventricular septal defect. As relative anaemia develops, a demand for increased left ventricular output occurs. This increases the work load on the left ventricle. and its oxygen requirement is increased. With anaemia there is a decrease in oxygen-carrying capacity of the blood, and the oxygen supply to the myocardium, which has an increased demand, may be inadequate, resulting in cardiac failure. 


\section{Pulmonary vasculature and ventricular septal defect}

Since the development of left-to-right shunt and increased pulmonary blood flow is so directly dependent on the decrease in pulmonary vascular resistance after birth, and as the major decline in pulmonary vascular resistance occurs within the 2-3 weeks after birth, it might be expected that cardiac failure would be apparent in this period in an infant with ventricular septal defect. It is, however, uncommon before 6-12 weeks after birth (Hoffman and Rudolph, 1965). This late development of failure may be explained on the fact that in infants with large ventricular septal defect the medial muscle layer in the small pulmonary vessels does not regress as rapidly as normal (Wagenvoort, 1962), and there is a slower decrease in pulmonary vascular resistance (Hoffman and Rudolph, 1965). The reason for this slower change in infants with ventricular septal defect is not known, but it may be a response to the higher pulmonary arterial pressures to which these vessels are subjected because of the large communication between the left and right ventricles.

An additional factor which may be important in affecting the normal pulmonary vascular changes after birth is persistent hypoxia, as occurs in infants born at high altitude, or when there is persistent pulmonary disease after birth. The effect of the hypoxia in maintaining a high pulmonary vascular resistance may influence the haemodynamic manifestation of ventricular septal defect after birth; thus cardiac failure of severe degree is less frequent in infants with ventricular septal defect born at high altitude (Vogel, McNamara, and Blount, 1967).

A drop in pulmonary vascular resistance which is more rapid than normal may also occur. We have observed that cardiac failure frequently occurs at an earlier stage after birth in premature infants than in mature infants (J. I. E. Hoffman, and A. M. Rudolph, unpublished observations). This may well be explained on the fact that the medial muscle layer in the pulmonary vessels of the immature foetus is less fully developed, and thus the premature infant at birth has pulmonary arterioles with less muscle. There may be a more rapid drop in pulmonary vascular resistance after birth with earlier onset of cardiac failure.

\section{Conclusions}

A ventricular septal defect, even of large size, is not likely to exert any major influence on the foetal circulation in view of the large communication between the aorta and pulmonary artery (the ductus arteriosus) that already exists.

The development of left-to-right shunt, increased pulmonary blood flow, and thus increased volume overload on the left ventricle in infants with large ventricular septal defect is dependent on the normal decrease in pulmonary vascular resistance and rise in systemic vascular resistance which occurs after birth.

The presence of a large ventricular septal defect in turn influences the normal postnatal changes in the pulmonary circulation and delays the decrease in pulmonary vascular resistance.

Slower decline in pulmonary vascular resistance as produced by hypoxia, and more rapid decline as occurs in premature infants, may influence the clinical features when a large ventricular septal defect is present.

\section{References}

Campbell, A. G. M., Dawes, G. S., Fishman, A. P., Hyman, A. I., and Perks, A. M. (1968). The release of a bradykinin-like pulmonary vasodilator substance in foetal and new-born lambs. fournal of Physiology, 195, 83.

Cassin, S., Dawes, G. S., Mott, J. C., Ross, B. B., and Strang, L. B. (1964). The vascular resistance of the foetal and newly ventilated lung of the lamb. fournal of Physiology, 171, 61.

Heymann, M. A., Rudolph, A. M., Nies, A. S., and Melmon, K. L. (1969). Bradykinin production associated with oxygenation of the fetal lamb. Circulation Research, 25, 521.

Hoffman, J. I. E., and Rudolph, A. M. (1965). The natural history of ventricular septal defects in infancy. American fournal of Cardiology, 16, 634.

Lloyd, T. C. (1969). In The Pulmonary Circulation and Interstitial Space, p. 2878. Ed. by A. P. Fishman and H. H. Hecht. University of Chicago Press, Chicago.

Reynolds, S. R. M. (1956). The fetal and neonatal pulmonary vasculature in the guinea pig in relation to hemodynamic changes at birth. American fournal of Anatomy, 98, 97.

Rudolph, A. M. (1965). The effects of postnatal circulatory adjustments in congenital heart disease. Pediatrics, 36, 763.

- Drorbaugh, J. E., Auld, P. A. M., Rudolph, A. J., Nadas, A. S., Smith, C. A., and Hubbell, J. P. (196r). Studies on the circulation in the neonatal period. The circulation in the respiratory distress syndrome. Pediatrics, 27, 551.

, and Heymann, M. A. (1967). The circulation of the fetus in utero. Methods for studying distribution of blood flow, cardiac output and organ blood flow. Circulation Research, 21, 163.

$\longrightarrow$, and Yuan, S. (1966). Response of the pulmonary vasculature to hypoxia and $\mathrm{H}^{+}$ion concentration changes. Fournal of Clinical Investigation, 45, 399.

Vogel, J. H. K., McNamara, D. C., and Blount, S. G., Jr. (1967). Role of hypoxia in determining pulmonary vascular resistance in infants with ventricular septal defects. American fournal of Cardiology, 20, 346.

Wagenvoort, C. A. (1962). The pulmonary arteries in infants with ventricular septal defect. Medicina Thoracalis, 19, 354. 